\title{
Interdisziplinäres Update der urogenitalen Diagnostik
}

Das Symposium der AG Uroradiologie der DRG, das vom 20. bis 21. Oktober 2017 in Düsseldorf stattfindet, steht im Zeichen der interdisziplinären urogenitalen Diagnostik. Experten der Radiologie, Urologie und Gynäkologie erläutern nicht nur Neuerungen und Standards der bildgebenden Diagnostik, sondern diskutieren auch Anforderungen an ein gemeinsames individuelles Therapiemanagement. Die beiden wissenschaftlichen Leiter der Veranstaltung, Priv.-Doz. Dr. med. Lars Schimmöller und Dr. med. Céline Alt-Radtke, erläutern im Interview, weshalb Sie in diesem Jahr die interdisziplinäre Zusammenarbeit in den Mittelpunkt rücken und welche Themen die Teilnehmer darüber hinaus erwartet.

Die Uroradiologie beschäftigt sich mit vielen Organen und Organsystemen. Welche Bereiche stehen denn aktuell besonders im Fokus von Forschung und Wissenschaft?

Priv.-Doz. Dr. Schimmöller In der Tat ist die Uroradiologie und die Urogenitaldiagnostik in viele Fachgebiete involviert, was diesen Teilbereich der Radiologie äußerst spannend und vielseitig macht. Sie ist fester Bestandteil diverser Leitlinien und Diagnostikpfade und unterliegt somit auch einer stetigen Aktualisierung und Optimierung. Die sicherlich größten Entwicklungen der letzten Jahre erfolgten in der Prostatadiagnostik mit dem immensen Potential der multiparametrischen MRT und den MR-gestützten Biopsieverfahren, aber auch der Tracer-basierten Diagnostik, z. B. mittels des prostataspezifischen Membranantigens (PSMA).

Frau Dr. Alt-Radtke Der wissenschaftliche Fokus der gynäkologischen Uroradiologie liegt besonders auf der Optimierung der funktionellen MRT in der Tumordiagnostik, insbesondere hinsichtlich der Diffusionsbildgebung und der Lymphknotendiagnostik. Ein sehr wichtiges Thema, das mittlerweile auch in der klinischen Routine Einzug gehalten hat, ist die Beckenbodendiagnostik mit dynamischer MRT, da immer mehr Menschen mit symptomatischen und oft-

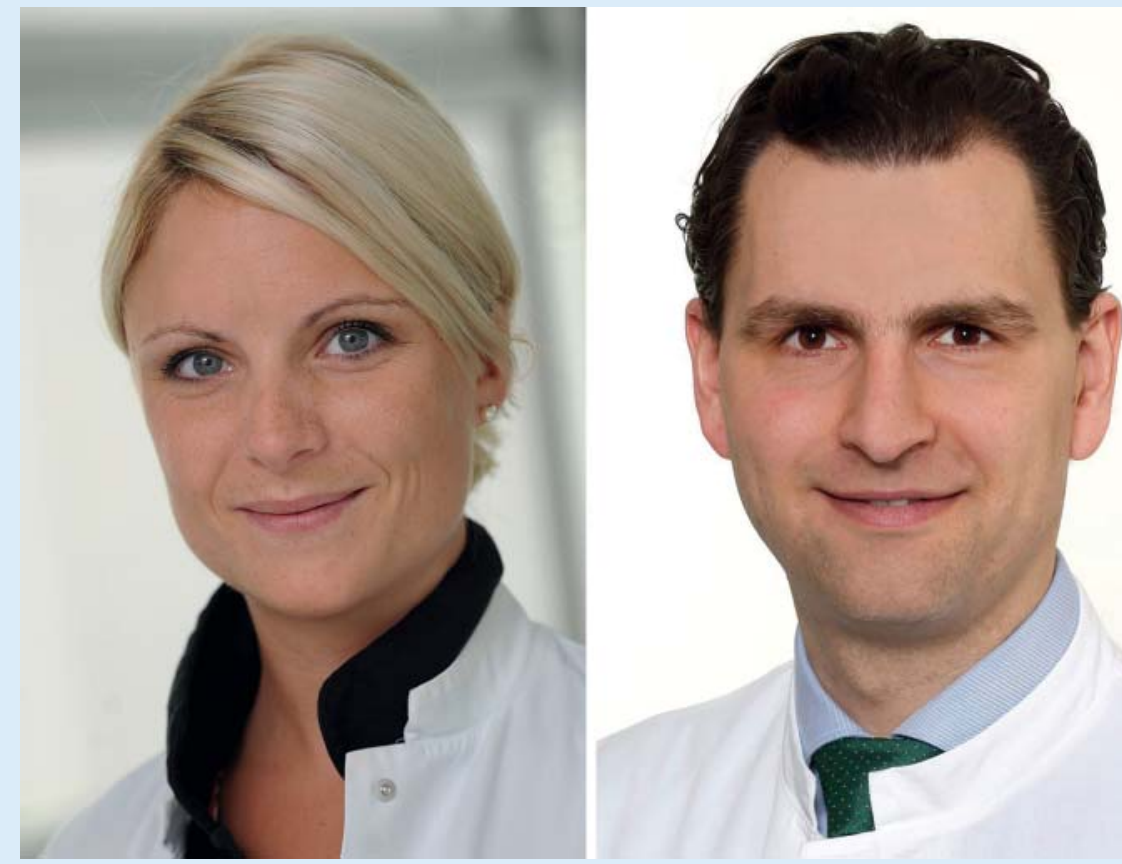

Dr. Céline Alt-Radtke und Priv.-Doz. Dr. Lars Schimmöller, wissenschaftliche Leiter des Urologischen Symposiums 2017 „Interdisziplinäre Urogenitale Diagnostik“

mals komplexen Senkungszuständen nach einer umfassenden Diagnostik fragen. Aber auch der Beckenboden ist in seiner Beschaffenheit und Funktion noch nicht vollständig erforscht und steht damit seit Jahren im Fokus urologischer, gynäkologischer und radiologischer Forschung.

Was zeichnet Ihrer Meinung nach eine gute uroradiologische Bildgebung aus?

Priv.-Doz. Dr. Schimmöller Wesentliche Voraussetzung für eine gute Bildgebung sind natürlich qualifizierte klinische Informationen über die Patientin oder den Patienten sowie eine möglichst präzise Darstellung des medizinischen Problems bzw. der Fragestellung. Ziel der Bildgebung sollte es dann sein, eine möglichst exakte Antwort hierauf zu geben und bei unklaren Fällen entsprechende Lösungen anzubieten. Die Untersuchungen sollten daher dem aktuellen Standard der radiologischen Diagnostik entsprechen, die im Falle der MRT neben hochaufgelösten und funktionellen Sequenzen gerade bei der gynäkologischen Bildgebung organspezifische Kippungen enthält. Eine immer schnellere, möglichst kostensparende, präzise, sichere, sowie breit verfügbare Diagnostik ist natürlich eine stete Anforderung an die Bildgebung, nichtsdestotrotz müssen die medizinische und biologische Heterogenität sowie die gegenwärtigen Möglichkeiten für Routineuntersuchungen berücksichtigt werden.

Welche Rolle spielt die Radiologie in der interdisziplinären Zusammenarbeit bzw. für das individuelle Therapiemanagement?

Frau Dr. Alt-Radtke Hinsichtlich eines individuellen Therapiemanagements ist die strukturierte und standardisierte Befundung von großer Wichtigkeit. Systematische Diagnostikpfade erleichtern dabei die interdisziplinäre Zusammenarbeit. Die Radiologie ist ein wichtiger klinischer Partner, der durch eine objektive und reproduzierbare Bildgebung den interdisziplinären Gedankenaustausch anregt und eine sinnvolle Ergänzung zur Erarbeitung individueller Therapiekonzepte liefert. Dies spiegelt sich unter anderem auch darin wieder, dass die organbezogene lokale Bildgebung mittlerweile ein fester Bestandteil in den Leitlinien ist. 
Das diesjährige Uroradiologische Symposium steht ganz im Zeichen der interdisziplinären Zusammenarbeit. Welche Themenschwerpunkte dürfen die Teilnehmer erwarten?

Priv.-Doz. Dr. Schimmöller Die nichtradiologischen Fachgebiete mit dem größten Anteil an urogenitaler Diagnostik sind die Urologie und die Gynäkologie. Aus diesem Grund haben wir auch Experten aus diesen beiden Gebieten eingeladen, um mit ihnen gemeinsam die Anforderungen und aktuellen Möglichkeiten der Bildgebung darzustellen und zu diskutieren. Themenschwerpunkte sind neben dem Update der Nierenund Harnblasendiagnostik die Abklärung gynäkologischer Tumoren (Uterus, Zervix, Ovar, Vulva und Vagina), die Beckenbodenund Inkontinenzdiagnostik sowie der aktuelle Stand und Perspektiven der modernen Prostatadiagnostik. Ferner wird eine Vor- tragssession die pädiatrische urogenitale Diagnostik und die Diagnostik und Therapie von Hodentumoren beleuchten.

Weshalb sollte man aus Ihrer Sicht die Veranstaltung auf gar keinen Fall verpassen?

Frau Dr. Alt-Radtke Das diesjährige Uroradiologische Symposium in Düsseldorf ermöglicht ein umfassendes und interdisziplinäres Update der urogenitalen Diagnostik mit Freiraum zur Diskussion und der Möglichkeit eines fächerübergreifenden Austausches zu den uroradiologischen Themengebieten. Es werden die aktuellen Leitlinien sowie moderne, innovative und auch zukünftige Ansätze der Bildgebung fundiert erarbeitet.

Priv.-Doz. Dr. Lars Schimmöller Diese Veranstaltung soll die zukunftsorientierte und enge Zusammenarbeit innerhalb der verschiedenen Fachbereiche stärken. Im Vordergrund steht deshalb der Erfahrungsund Wissensaustausch, der dabei helfen kann, das eigene diagnostische und therapeutische Patientenmanagement weiter zu verbessern.

Vielen Dank für das Gespräch!

Uroradiologisches Symposium 2017 Interdisziplinäre Urogenitale Diagnostik 20./21. Oktober 2017, Düsseldorf Informationen zum Programm und zur Anmeldung finden Sie hier (LINK http://www.drg.de/de-DE/178/veranstaltungskalender/show/1407/Urologisches-Symposium-2017-Interdisziplinaere-Urogenitale-Diagnostik) 